\title{
Excitation Modes of Bright Matter-Wave Solitons
}

\author{
Andrea Di Carli, Craig D. Colquhoun, Grant Henderson, Stuart Flannigan, Gian-Luca Oppo, \\ Andrew J. Daley, Stefan Kuhr, and Elmar Haller@ \\ Department of Physics and SUPA, University of Strathclyde, Glasgow G4 ONG, United Kingdom
}

(Received 2 May 2019; revised manuscript received 18 July 2019; published 17 September 2019)

\begin{abstract}
We experimentally study the excitation modes of bright matter-wave solitons in a quasi-one-dimensional geometry. The solitons are created by quenching the interactions of a Bose-Einstein condensate of cesium atoms from repulsive to attractive in combination with a rapid reduction of the longitudinal confinement. A deliberate mismatch of quench parameters allows for the excitation of breathing modes of the emerging soliton and for the determination of its breathing frequency as a function of atom number and confinement. In addition, we observe signatures of higher-order solitons and the splitting of the wave packet after the quench. Our experimental results are compared to analytical predictions and to numerical simulations of the one-dimensional Gross-Pitaevskii equation.
\end{abstract}

DOI: 10.1103/PhysRevLett.123.123602

The dispersionless propagation of solitary waves is one of the most striking features of nonlinear dynamics, with multiple applications in hydrodynamics, nonlinear optics, and broadband long-distance communications [1]. In fiber optics, one-dimensional (1D) "bright" solitons, i.e., solitons presenting a local electric field maximum with onedimensional propagation, have been observed [2]. They exhibit a dispersionless flow and excitation modes such as breathing or higher-order modes [2-4]. Matter waves can also display solitary dispersion properties. Typically, bright matter-wave solitons are created in quasi-1D systems by quenching the particle interaction in a Bose-Einstein condensate (BEC) from repulsive to attractive [5]. Recent experiments demonstrated the collapse [6], collisions [7], reflection from a barrier [8], and the formation of trains [9-11] of bright solitons.

In this Letter, we experimentally study the excitation modes of a single bright matter-wave soliton. In previous studies, other dynamical properties have been observed, such as the center-of-mass oscillation of solitons in an external trap [7] and excitations following the collapse of attractive BECs $[6,12]$. Here, we probe the fundamental breathing mode of a single soliton by measuring its oscillation frequency and the time evolution of its density profile. In addition, we observe signatures of higher-order matter-wave solitons, which can be interpreted as stable excitations with periodic oscillations of the density profile and phase, or as a bound state of overlapping modes [3,13].

Published by the American Physical Society under the terms of the Creative Commons Attribution 4.0 International license. Further distribution of this work must maintain attribution to the author(s) and the published article's title, journal citation, and DOI.
The shape-preserving evolution of a matter-wave soliton is due to a balancing of dispersive and attractive terms in the underlying 3D Gross-Pitaevskii equation (GPE) [14]. For quasi-1D systems with tight radial confinement, we can approximate the matter wave in the 3D GPE by the product of a Gaussian wave function for the radial direction and a function $f(z)$ for the longitudinal direction (see Ref. [15]). Depending on the ansatz for the Gaussian with either constant or varying radial sizes, $f(z)$ satisfies either the 1D GPE or the nonpolynomial Schrödinger equation [18]. We make reference to the analytical solutions of the 1D GPE in the Letter, but use both equations in our numerical simulations [15].

For the 1D GPE, an ansatz for the normalized longitudinal wave function $f(z)$ is of the form

$$
f(z)=\frac{1}{\sqrt{2 l_{z}}} \operatorname{sech}\left(\frac{z}{l_{z}}\right)
$$

with a single parameter $l_{z}$ that determines both the longitudinal size and the amplitude of the soliton. Solitons form with a value of $l_{z}$ that minimizes the total energy and that provides a compromise between the kinetic and the interaction energies. This is illustrated in Fig. 1(b), which shows the energy of the wave packet for varying sizes $l_{z}$ [19]. The kinetic energy provides a potential barrier for small $l_{z}$ that prevents the collapse of the soliton, while its spreading is inhibited by the interaction energy, which increases for large $l_{z}$.

Even without an external longitudinal potential, the soliton is stable against small perturbations of $l_{z}$. In a way, a bright matter-wave soliton creates its own trapping potential, which defines its size and excitation modes. Variational methods provide accurate predictions of its size 

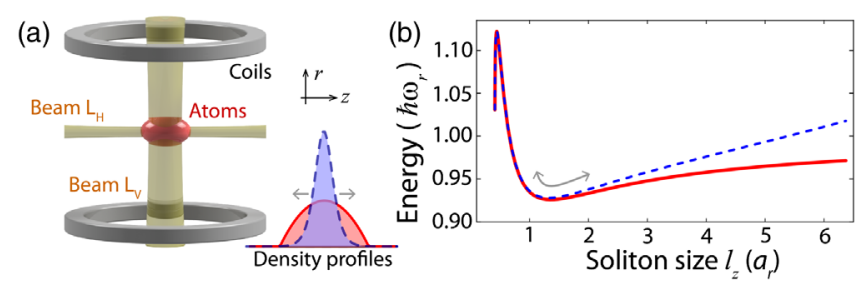

(c)
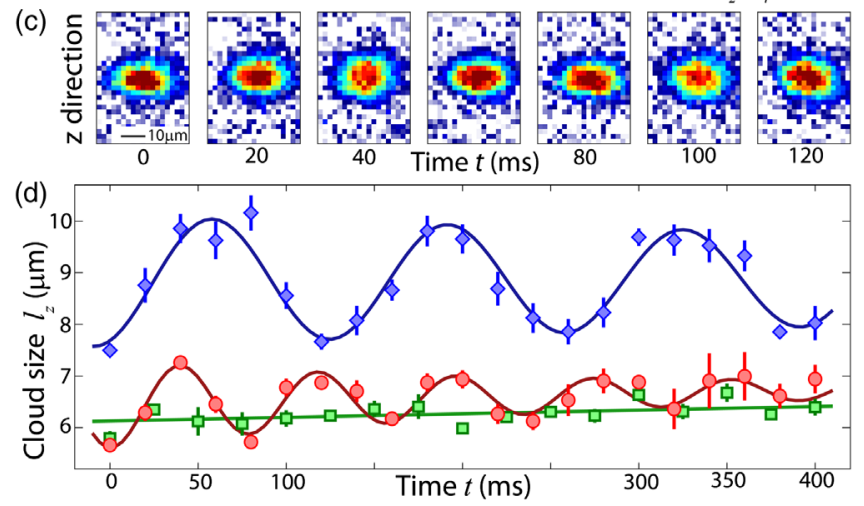

FIG. 1. Experimental setup and oscillation measurements. (a) Sketch of the experimental setup. Inset: Density profiles for a BEC (solid red line) and for a soliton (dashed blue line). (b) Total energy of a soliton, $a=-5.2 a_{0}, \omega_{r}=2 \pi \times 95 \mathrm{~Hz}$, $N=2000$, with an external trap, $\omega_{z}=2 \pi \times 5 \mathrm{~Hz}$ (dashed blue line), and without external trap, $\omega_{z}=0 \mathrm{~Hz}$ (solid red line). (c) Absorption images after a free-expansion time of $16 \mathrm{~ms}$ [from dataset with circles in (d)], integrated density profile for $t=$ $60 \mathrm{~ms}$ (blue line) and fit (dashed red line). (d) Oscillations of a quantum gas after the quench procedure. Blue diamonds, quench of only $\omega_{z}$ for a BEC $(Q 1)$; red circles, additional interaction quench to create soliton $(Q 3)$; green squares, optimized quench parameters to minimize breathing of the soliton $(Q 2)$. Uncertainty intervals indicate \pm 1 standard error.

at the energy minimum which can be calculated analytically $[13,20]$ or numerically [19]. For the fundamental solution (order $n=1$ ) of the 1D GPE with an atom number $N, s$-wave scattering length $a$, and radial trapping frequency $\omega_{r}$, the size $l_{z}$ corresponds to the healing length at the peak density of the soliton, i.e., $l_{z}^{(n=1)}=a_{r}^{2} /(N|a|)$ $[13,19]$. Here, $a_{r}=\sqrt{\hbar /\left(m \omega_{r}\right)}$ is the radial harmonic oscillator length. Small deviations of $l_{z}$ close to the energy minimum lead to oscillations of the soliton size. We use those oscillations resulting from an initial mismatch of $l_{z}$ to experimentally measure the self-trapping frequency of the soliton potential.

Our experimental starting point is a Bose-Einstein condensate of 500-2000 cesium (Cs) atoms in the state $\left|F=3, m_{F}=3\right\rangle$ at scattering length of $a=+7 a_{0}$, where $a_{0}$ is Bohr's radius. The BEC is levitated by a magnetic field gradient, and it is confined by an optical dipole trap formed by the horizontal and vertical laser beams $L_{H}$ and $L_{V}$ [Fig. 1(a)]. An additional magnetic offset field allows us to tune the scattering length by means of a broad magnetic Feshbach resonance [21]. Details about our experimental setup, the levitation scheme, and the removal of atoms can be found in Refs. [15,22].
Our matter-wave solitons are confined to a quasi-1D geometry with almost free propagation along the horizontal direction and strong radial confinement of $\omega_{r}=$ $2 \pi \times 95 \mathrm{~Hz}$ provided by laser beam $L_{H}$. They are generated with a quench of the scattering length towards attractive interaction $\left(a_{i} \rightarrow a_{f}\right)$, and by a reduction of the longitudinal trap frequency $\left(\omega_{z, i} \rightarrow \omega_{z, f}\right)$. When changing $a$ and $\omega_{z}$ independently, the quenches excite inward and outward motions, respectively. Usually, it is desirable to minimize the excitations of the soliton by matching the initial Thomas-Fermi density profile of the BEC closely to the density profile of the soliton [inset of Fig. 1(a)]. However, we deliberately mismatch the quench parameters to create breathing oscillations of the soliton in order to study its self-trapping potential. Quenches with different parameters are labeled by the symbols $Q 1-Q 7$ (see Ref. [15]). Following an evolution time $t$ in quasi-1D and after a short period of $16 \mathrm{~ms}$ of expansion in free space, we take absorption images to determine the density profile of the atoms [Fig. 1(c)]. The cloud size $l_{z}(t)$ is determined by fitting the function $A(\operatorname{sech}(z / B))^{2}$ to the integrated 1D-density profiles with fit parameters $A$ and $B$ [15].

The response of the atomic cloud to the different quenches is presented in Fig. 1(d). We first quench only the longitudinal confinement by $25 \%$ to $\omega_{z, f}=2 \pi \times 4.3(2) \mathrm{Hz}$ (quench $Q 1$ in Ref. [15]) while keeping the repulsive interaction strength constant [Fig. 1(d), diamonds]. The BEC starts an outwards motion with an oscillation frequency of $2 \pi \times 7.5(1) \mathrm{Hz} \approx \sqrt{3} \omega_{z, f}$ as expected for a BEC in the Thomas-Fermi regime [23,24]. In a second measurement, we additionally quench the interaction strength $a_{f}$ to $-5.4 a_{0}$ and increase $\omega_{z, i}$ to match the initial size of the BEC to the expected size of the soliton [Q2, Fig. 1(d), squares]. As a result, we observe almost dispersionless solitons with a linear increase of the cloud size of 0.7(3) $\mu \mathrm{m} / \mathrm{s}$ [Fig. 1(d), green line]. Finally, we deliberately mismatch the initial size of the BEC by reducing $\omega_{z, i}(Q 3)$, and generate small-amplitude oscillations of the soliton with a frequency $\omega_{\text {sol }}$ of $2 \pi \times$ 12.8(4) Hz [Fig. 1(d), circles]. This breathing frequency of the soliton is significantly larger than any breathing frequency of a BEC or of noninteracting atoms, $2 \omega_{z, f}=$ $2 \pi \times 8.6(3) \mathrm{Hz}$. We observe no discernible oscillation in the radial direction after the quenches.

In a second experiment, we demonstrate that the breathing frequency $\omega_{\text {sol }}$ depends on the interaction term $\mathrm{Na}$ in the 1D GPE, a property typical of the nonlinear character of the soliton. We choose to change $N$, since the initial removal process is independent of the interaction quench, and we can study $\omega_{\text {sol }}$ without changing the quench protocol [ $Q 4$, Fig. 2(a), circles]. The measured values of $\omega_{\text {sol }}$ decrease for lower $N$, and they approach the breathing frequency $2 \omega_{z, f}$ for noninteracting atoms in a harmonic trap [Fig. 2(a), dashed line].

We compare our experimental data points to two theoretical models. In a numerical simulation of the 1D 


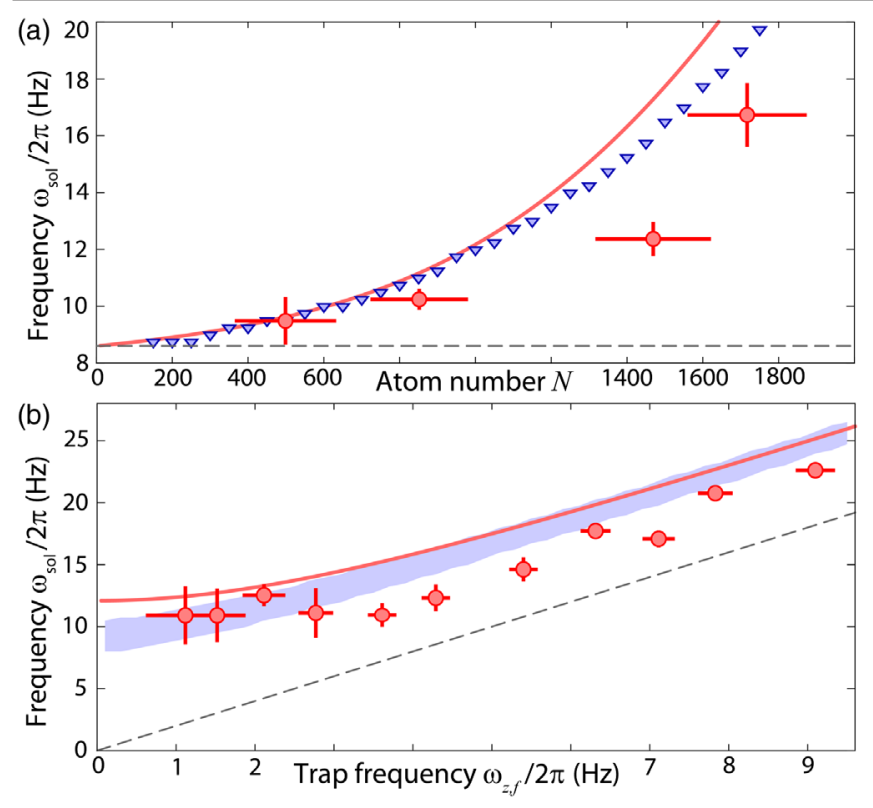

FIG. 2. Breathing frequency $\omega_{\text {sol }}$ of the soliton. (a) Atom number dependence $(Q 4)$. Red circles, experimental data; the uncertainty bars for the atom number indicate the standard deviation of $N$ over the first $100 \mathrm{~ms}$ of each frequency measurement. Blue triangles, simulation of the 1D GPE [15]. Red line, analytical approximation $[13,15]$. Dashed gray line, oscillation frequency of a noninteracting gas, $2 \omega_{z, f}$. (b) Dependence of $\omega_{\text {sol }}$ on the trap frequency (Q5). Red circles, experimental data points for $N \approx 1450$. Blue area, simulation of the $1 \mathrm{D}$ GPE for $N=1300-1500$. Red line, analytical approximation. Dashed gray line, $2 \omega_{z, f}$.

GPE, we use the ansatz in Eq. (1) to set the starting conditions, and we determine the breathing frequency from a spectral analysis of the time evolution of the wave function [15] [Fig. 2(a), triangles]. In addition, we use an analytical approximation for the breathing frequency (red line) calculated with a Lagrangian variational analysis at the energy minimum of the 3D GPE $[13,15]$. We find that both models agree well with the trend of the measurements of $\omega_{\text {sol }}$, although our experimental data points are systematically lower for large $N$ than our theoretical predictions. We speculate that this is due to nonharmonic contributions to the energy of the soliton on the breathing oscillations for finite oscillation amplitudes [Fig. 1(b)].

To determine the influence of the trapping potential, we measure the variation of $\omega_{\text {sol }}$ as we reduce the longitudinal trapping frequency $\omega_{z, f}(Q 5)$. Two regimes of $\omega_{\text {sol }}$ can be identified in Fig. 2(b) for varying the values of $\omega_{z, f}$. For large values of $\omega_{z, f}$, the trap dominates the breathing of the soliton and $\omega_{\text {sol }}$ increases like $2 \omega_{z, f}$. For small values of $\omega_{z, f}$, interactions dominate the breathing of the soliton and $\omega_{\text {sol }}$ reaches a constant value. This offset of the breathing frequency is a result of the "self-trapping" potential of a free soliton.

Again, we compare the experimental results with our theoretical model [Fig. 2(b), red line] and the numerical simulations of the 1D GPE. The blue band in Fig. 2(b) indicates the simulated frequencies for $N=1300$ to $N=1500$. The simulation predicts a lower breathing frequency for the free soliton than the analytical approximation, but all curves are within the uncertainly range of the experimental data.

External trapping potentials can in principle alter the soliton dynamics [7,25,26], causing, e.g., modulations of the soliton's tails due to residual nonautonomous terms of the 1D GPE in a harmonic potential [27]. For the following experiments, however, we employ trap frequencies that are significantly smaller than the observed oscillation frequencies of the soliton $\left(2 \omega_{z}<\omega_{\text {sol }}\right)$ to decouple the influence of the trapping potential. In summary, for small-amplitude oscillations we find good agreement of $\omega_{\text {sol }}$ between our experimental results and analytical and numerical predictions based on the 1D GPE (and nonpolynomial Schrödinger equation [15]).

Breathing oscillations of $l_{z}$ close to the equilibrium size are not the only possible excitation modes of solitons. The existence of higher-order solitons has been predicted in the nonlinear Schrödinger equation [3], and has been observed for optical solitons in silica-glass fibers $[2,4]$. A soliton of order $n$ can be interpreted as a bound state of $n$ strongly overlapping solitons [13]. By exploiting the equivalence of the nonlinear Schrödinger equation and 1D GPE, similar effects were later proposed for bright matter-wave solitons $[13,28]$, where it was suggested that $n$ th-order solitons can be generated by a rapid increase of the attractive interaction strength by a factor $n^{2}$. Similarly, our simulations of the 1D GPE show that higher-order solitons can be created for an increased initial size of the wave packet. An $n$ th-order soliton forms for a sech-shaped wave function with an initial size $l_{z}^{(n)}$ that is the $n^{2}$ multiple of the healing length $l_{z}^{(1)}$, i.e., $l_{z}^{(n)}=n^{2} l_{z}^{(1)}[15]$.

Within the 1D GPE theory, both creation methods result in the periodic development of multipeaked structures for higher-order solitons [3,29]; e.g., they create a sharp central peak with side wings for a second-order soliton [Fig. 3(a)] and a double peak for a third-order soliton [15]. Sizes and interaction quenches that do not fulfil the previous conditions lead to a "shedding" of the atomic density in the $z$ direction. The wave packet oscillates and loses particles until its size and shape match the next (lower $n$ ) higherorder soliton [3]. For a second-order soliton, the predicted oscillation period $T^{(2)}$ is [13]

$$
T^{(2)}=\frac{8 \pi}{\hbar} m\left(\frac{a_{r}^{2}}{N\left|a_{f}\right|}\right)^{2} .
$$

Recently, excitation modes of higher order have also been used as a test bed for various theoretical models beyond GP theory. The fragmentation of solitons with an increased initial width was predicted within the multiconfigurational time-dependent Hartree method for bosons 

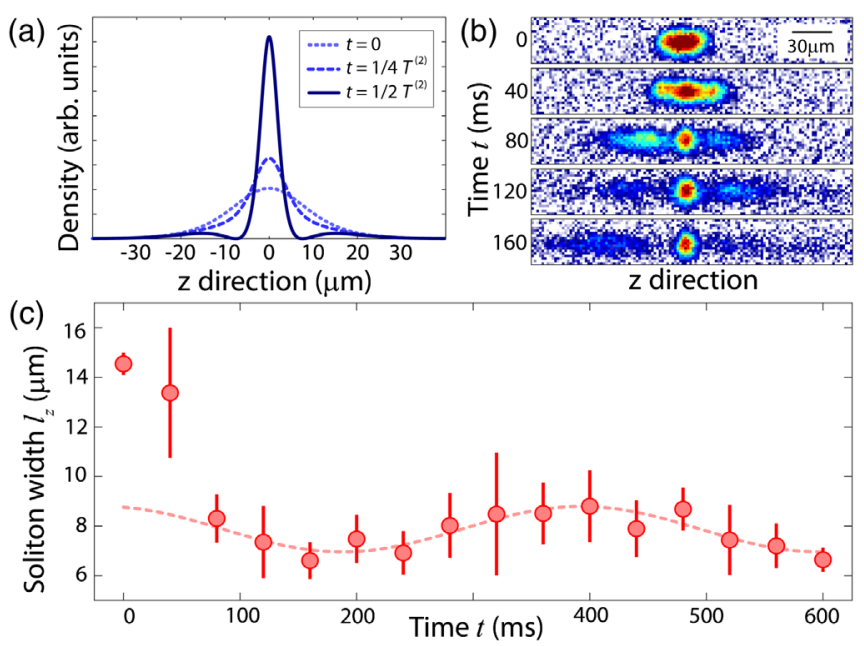

FIG. 3. Time evolution after a strong quench of interactions and trap frequency (Q6). (a) 1D GPE simulation of the density profiles for a second-order soliton with 1100 atoms, $a_{f}=-5.3 a_{0}$, and with an oscillation period $T^{(2)}$ of $432 \mathrm{~ms}$. (b) Absorption images at time $t$ after the quench and after $11 \mathrm{~ms}$ of free expansion. (c) Time evolution of the measured width $l_{z}$ of the central wave packet (red circles), sinusoidal fit with period 420(30) ms (dashed red line). The uncertainty intervals indicate \pm 1 standard deviation.

[30] and critically discussed [31], and the influence of quantum effects on the dissociation process was investigated [32-34].

Here, we apply two different quench protocols to study the evolution of strongly excited solitons. Depending on the initial size and the quench parameters, we observe shedding and fragmentation of the wave packet, and we measure oscillation frequencies that indicate the creation of higherorder solitons. To demonstrate the effect of a strong quench of an elongated BEC, we increase $a_{i}$ and reduce $\omega_{z, i}$ before ramping $a$ and $\omega_{z}$ to $-5.3 a_{0}$ and $2 \pi \times 0.0(6) \mathrm{Hz}$ in $13 \mathrm{~ms}$ (Q6). Our quench induces an initial spreading of the wave packet, followed first by a strong shedding of atoms, and then by the formation of a soliton that contains approximately $1 / 3$ of the initial atom number [Fig. 3(b)]. We determine the soliton width and find a slow oscillation of $l_{z}(t)$ with a frequency of $2 \pi \times 2.4(2) \mathrm{Hz}$ [Fig. 3(c)]. This frequency is significantly smaller than the expected breathing frequency of first-order solitons, $2 \pi \times 6.0 \mathrm{~Hz}$, and it matches well to the expected frequency of $2 \pi \times 2.3 \mathrm{~Hz}$ for second-order solitons in Eq. (2).

Observing shedding and oscillations agrees with the predictions for higher-order solitons within the 1D GPE [3]; however, we find a strong dependence on details of the quench protocol and on the dynamical evolution during the quench. For a closer match to theoretical works [13], we implement a double-quench protocol, with a first quench to generate a soliton with weak attractive interaction, $a_{f}=-0.8 a_{0}, \omega_{f}=2 \pi \times 1.4(2) \mathrm{Hz}$, and, after a settling time of $25 \mathrm{~ms}$, a second quench of only the interaction

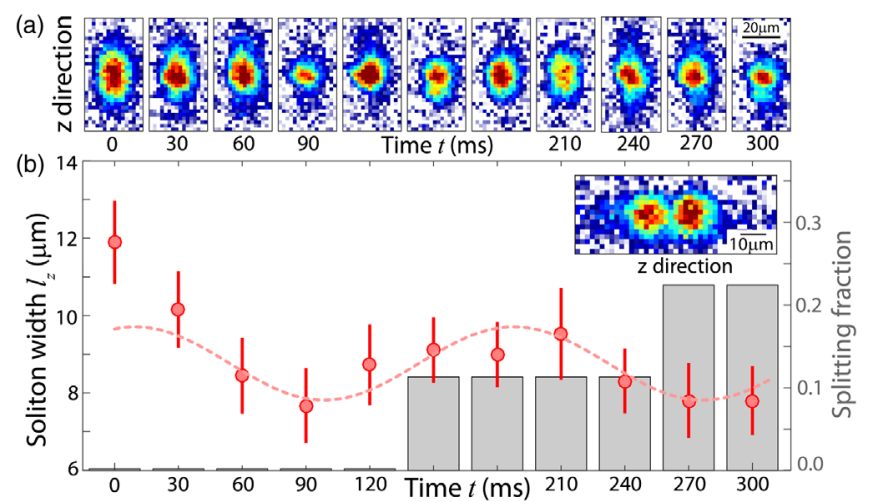

FIG. 4. Second-order soliton and splitting after the double quench $Q 7$. (a) Absorption images at time $t$ after the quench and after $7 \mathrm{~ms}$ of free expansion. (b) Time evolution of the measured width $l_{z}$ of the central wave packet (red circles), sinusoidal fit with period 180(20) ms (dashed red line). The expected period from the 1D GPE simulations is $192 \mathrm{~ms}$. The histogram counts the fraction of images showing a splitting of the wave function (9 repetitions per time step). Inset: Absorption image of a split matter wave for $t=210 \mathrm{~ms}$.

strength, $a_{f}=-4.6 a_{0}(Q 7)$. Starting with approximately 2200 atoms, we observe no shedding but a small loss of 300 atoms during the first $60 \mathrm{~ms}$. The vertical density profiles in our absorption images [Fig. 4(a)] resemble the expected profiles of a second-order soliton [Fig. 3(a)], and the vertical width of the wave packet oscillates with a frequency of $2 \pi \times 5.6(6) \mathrm{Hz}$ [Fig. 4(b)], which matches the expected frequency of $2 \pi \times 5.2 \mathrm{~Hz}$ for second-order solitons $(2 \pi \times 13.2 \mathrm{~Hz}$ for the first-order solitions).

For both measurements [Figs. 3(c) and 4(b)], a small percentage of absorption images show a splitting of the soliton into two fragments [inset of Fig. 4(b)], and they are omitted from the fitting procedure. Because of the destructive nature of our absorption images it is difficult to conclude on the evolution and on the cause of the splitting process. A double-peak structure in the density profile can indicate the generation of a third-order soliton, fragmentation due to quantum effects, or simply an insufficient technical control of our quench parameters. For our setup, the control of horizontal magnetic field gradients to avoid longitudinal accelerations is especially challenging [22]. The percentage of images that show a splitting of the wave packet increases for longer evolution times, and we indicate their fraction in Fig. 4(b) with a histogram.

In conclusion, we experimentally studied the creation and the excitation of breathing modes of bright matterwaves solitons in a quasi-one-dimensional geometry after a quench of interaction and longitudinal confinement. We measured the "self-trapping" frequency $\omega_{\text {sol }}$ for first-order solitons and its dependence on $N$ and $\omega_{z}$. For stronger excitations and for a double-quench protocol, we observed signatures of second-order solitons and the shedding and splitting of the wave function. Further measurements of the 
splitting process and the damping of the oscillations due to shedding are necessary to distinguish technical fluctuations from higher-order solitons and fragmentation due to quantum effects [32-34].

The data used in this publication are openly available at the University of Strathclyde KnowledgeBase [35].

We thank L. D. Carr for helpful initial discussions during his visit. We acknowledge support by the EU through "QuProCS" (GA 641277) and the ETN "ColOpt" (GA 721465), and by the EPSRC Programme Grant "DesOEQ" (EP/P009565/1). A. D. C. acknowledges financial support by EPSRC and SFC via the IMPP. G. H. acknowledges support from a Carnegie Undergraduate Vacation Scholarship.

[1] M. P. T. Dauxois, Physics of Solitons (Cambridge University Press, Cambridge, England, 2006).

[2] L. F. Mollenauer, R. H. Stolen, and J. P. Gordon, Phys. Rev. Lett. 45, 1095 (1980).

[3] J. Satsuma and N. Yajima, Prog. Theor. Phys. Suppl. 55, 284 (1974).

[4] R. H. Stolen, W. J. Tomlinson, and L. F. Mollenauer, Opt. Lett. 8, 186 (1983).

[5] L. Khaykovich, F. Schreck, G. Ferrari, T. Bourdel, J. Cubizolles, L. D. Carr, Y. Castin, and C. Salomon, Science 296, 1290 (2002).

[6] E. A. Donley, N. R. Claussen, S. L. Cornish, J. L. Roberts, E. A. Cornell, and C. E. Wieman, Nature (London) 412, 295 (2001).

[7] J. H. V. Nguyen, P. Dyke, D. Luo, B. A. Malomed, and R. G. Hulet, Nat. Phys. 10, 918 (2014).

[8] A. L. Marchant, T. P. Billam, T. P. Wiles, M. M. H. Yu, S. A. Gardiner, and S. L. Cornish, Nat. Commun. 4, 1865 (2013).

[9] K. E. Strecker, G. B. Partridge, A. G. Truscott, and R. G. Hulet, Nature (London) 417, 150 (2002).

[10] J. H. V. Nguyen, D. Luo, and R. G. Hulet, Science 356, 422 (2017)

[11] T. Mežnaršič, T. Arh, J. Brence, J. Pišljar, K. Gosar, Ž. Gosar, R. Žitko, E. Zupanič, and P. Jeglič, Phys. Rev. A 99, 033625 (2019).

[12] S. L. Cornish, S. T. Thompson, and C. E. Wieman, Phys. Rev. Lett. 96, 170401 (2006).

[13] L. D. Carr and Y. Castin, Phys. Rev. A 66, 063602 (2002).

[14] F. Dalfovo, S. Giorgini, L. P. Pitaevskii, and S. Stringari, Rev. Mod. Phys. 71, 463 (1999).

[15] See Supplemental Material at http://link.aps.org/ supplemental/10.1103/PhysRevLett.123.123602 for additional information, which includes Refs. [16,17].
[16] S. Cowell, H. Heiselberg, I. E. Mazets, J. Morales, V. R. Pandharipande, and C. J. Pethick, Phys. Rev. Lett. 88, 210403 (2002).

[17] P. J. Everitt, M. A. Sooriyabandara, M. Guasoni, P. B. Wigley, C. H. Wei, G. D. McDonald, K. S. Hardman, P. Manju, J. D. Close, C. C. N. Kuhn, S. S. Szigeti, Y. S. Kivshar, and N. P. Robins, Phys. Rev. A 96, 041601(R) (2017).

[18] L. Salasnich, A. Parola, and L. Reatto, Phys. Rev. A 65, 043614 (2002).

[19] N. G. Parker, S. L. Cornish, C. S. Adams, and A. M. Martin, J. Phys. B 40, 3127 (2007).

[20] D. Longenecker and E. J. Mueller, Phys. Rev. A 99, 053618 (2019).

[21] M. Berninger, A. Zenesini, B. Huang, W. Harm, H.-C. Nägerl, F. Ferlaino, R. Grimm, P. S. Julienne, and J. M. Hutson, Phys. Rev. A 87, 032517 (2013).

[22] A. D. Carli, C. D. Colquhoun, S. Kuhr, and E. Haller, New J. Phys. 21, 053028 (2019).

[23] C. Menotti and S. Stringari, Phys. Rev. A 66, 043610 (2002).

[24] E. Haller, M. Gustavsson, M. J. Mark, J. G. Danzl, R. Hart, G. Pupillo, and H.-C. Nägerl, Science 325, 1224 (2009).

[25] V. Serkin, A. Hasegawa, and T. Belyaeva, in Odyssey of Light in Nonlinear Optical Fibers: Theory and Applications, edited by K. Porsezian and R. Ganapathy (CRC Press, Boca Raton, FL, 2015), pp. 145-187.

[26] V. Serkin and T. Belyaeva, Optik (Stuttgart) 176, 38 (2019).

[27] C. H. Tenorio, E. V. Vargas, V. N. Serkin, M. A. Granados, T. L. Belyaeva, R. P. Moreno, and L. M. Lara, Quantum Electron. 35, 929 (2005).

[28] J. Golde, J. Ruhl, M. Olshanii, V. Dunjko, S. Datta, and B. A. Malomed, Phys. Rev. A 97, 053604 (2018).

[29] O. Gamayun and M. Semenyakin, J. Phys. A 49, 335201 (2016).

[30] A. I. Streltsov, O. E. Alon, and L. S. Cederbaum, Phys. Rev. Lett. 100, 130401 (2008).

[31] J. G. Cosme, C. Weiss, and J. Brand, Phys. Rev. A 94, 043603 (2016).

[32] C. Weiss and L. D. Carr, arXiv:1612.05545.

[33] V. A. Yurovsky, B. A. Malomed, R. G. Hulet, and M. Olshanii, Phys. Rev. Lett. 119, 220401 (2017).

[34] K. L. Ng, B. Opanchuk, M. D. Reid, and P. D. Drummond, Phys. Rev. Lett. 122, 203604 (2019).

[35] A. Di Carli, C. D. Colquhoun, G. Henderson, S. Flannigan, G.-L. Oppo, A. J. Daley, S. Kuhr, and E. Haller, Data for 'Excitation Modes of Bright Matter-Wave Solitons', University of Strathclyde KnowledgeBase, https://doi.org/ 10.15129/699a004b-fbf3-4f37-b2f3-9c1c97f44f0f. 